(C) 20XX IEEE. Personal use of this material is permitted. Permission from IEEE must be obtained for all other uses, in any current or future media, including reprinting/republishing this material for advertising or promotional purposes, creating new collective works, for resale or redistribution to servers or lists, or reuse of any copyrighted component of this work in other works.

Kara, Peter A., Cserkaszky, Aron, Darukumalli, Subbareddy, Barsi, Attila and Martini, Maria G. (2017) On the edge of the seat: reduced angular resolution of a light field cinema with fixed observer positions. IProceedings of the 2017 Ninth International Conference on Quality of Multimedia Experience (QoMEX). Piscataway, U.S. :

Institute of Electrical and Electronics Engineers, Inc. ISSN (online) 2472-7814 ISBN (electronic) 9781538640241.

https://doi.org/10.1109/QoMEX.2017.7965637 


\title{
On the Edge of the Seat: Reduced Angular Resolution of a Light Field Cinema with Fixed Observer Positions
}

\author{
Peter A. Kara*, Aron Cserkaszky ${ }^{\dagger \dagger}$, Subbareddy Darukumalli ${ }^{\dagger \ddagger}$, Attila Barsi ${ }^{\dagger}$, Maria G. Martini* \\ *WMN Research Group, Kingston University, London, UK \\ Email: \{p.kara, m.martini\}@kingston.ac.uk \\ ${ }^{\dagger}$ Holografika, Budapest, Hungary \\ Email: \{a.cserkaszky, s.darukumalli, a.barsi\}@holografika.com \\ $\ddagger$ Pazmany Peter Catholic University, Budapest, Hungary \\ Email: \{cserkaszky.aron, darukumalli.subbareddy\}@itk.ppke.hu
}

\begin{abstract}
The smooth motion parallax of light field displays is one of the most critical enablers of the glasses-free autostereoscopic 3D experience. This smoothness originates from an acceptable degree of angular resolution, which is derived from the number of views visualized in the given field of view. However, if human observers view the content without any movement, the requirement for angular resolution can be lower. In this paper, we introduce the results of a subjective quality assessment of visual content displayed on a light field cinema. The angular resolution of the content was different in each test condition, and participants were located in fixed positions during the experiment.
\end{abstract}

Keywords-Quality of Experience; Light Field Display; Angular resolution; Parallax effect; Subjective quality assessment

\section{INTRODUCTION}

In the recent years, the research on 3D light field visualization made Quality of Experience (QoE) concerns timely and relevant. This is particularly true since light field displays (LFDs) offer a high level of immersive, natural visual experience, compared to the currently commercially available solutions. Among the several factors that determine the subjective value of such displays, the smoothness of motion parallax definitely contributes a significant portion to the immersive and natural feeling. In practice, LFDs can be parallax both vertically and horizontally - depending on the solution of the display - but the horizontal parallax is more important due to typical conditions of viewing scenarios; users tend to have more movement left and right than up and down. After all, the Field of View (FOV) traditionally defines the horizontal angle interval in which the LFD was meant to be observed. Of course in order to achieve a parallax effect in a given direction, both the display and the content need to be parallax in that direction.

Visualization on LFDs can be characterized by several attributes (e.g., spatial resolution of content, depth values, Region of Interest (ROI) etc.), but angular resolution is the one chiefly responsible for the perceivable parallax effect. Angular resolution is defined to be the ratio of FOV and the number of views in the FOV, but dividing vice versa makes sense as well. If e.g., we have a FOV of 45 degrees and 90 views, the first definition states that there is a view every 0.5 degree, while the latter determines 2 views per degree. This is the angular resolution of content visualization - where views refer to the source that is to be converted - and is not to be confused with the angular resolution of the display itself, which is given by the minimal angle of change that rays can reproduce with respect to a single point on the screen [1].

Horizontal parallax can be experienced without any horizontal movement of the human observer, due to the horizontal separation of the 2 eyes of the person. Also, when one is sitting or standing in a given position with a given orientation, the movement of the eye already establishes the parallax effect, and this is accompanied by the fact that there is always a measurable extent of horizontal movement of one's head. However, this extent is not comparable with an actual movement in front of the screen of a display. As more motion enables a more intense experience of horizontal motion parallax, this can also assume a higher sensitivity towards the disturbances in the smoothness of the parallax effect (i.e., the crosstalk effect or the visible discrete image borders) during $3 \mathrm{D}$ visualization. If we approach this from the opposite direction, the requirements for the smoothness of horizontal parallax can be lower if the observer is not moving.

The research question investigated by this paper focuses on the angular resolution of visual content on LFDs. In particular, we study the QoE of 3D visualization on a light field cinema system with reduced angular resolution, and fixed observer position - just as in a real cinematic use case scenario, where people watch the entire content from one given seat. Our hypothesis is that the lack of observer movement could allow higher tolerance against low angular resolution, as the perceptual phenomenon of horizontal parallax plays a smaller role in such scenario. This paper presents the results of a subjective quality assessment experiment, where test participants rated the visual experience and acceptance of different still, rendered models visualized on a light field cinema system.

The remainder of this paper is structured as follows: Section II briefly introduces the related work in the research area. The details of the experimental setup are presented in Section III, followed by the results in Section IV. The paper is concluded in Section V, also pointing out potential continuations of the investigated topic. 


\section{RELATED WORK}

The work of Tamboli et al. [2] introduces the results of a subjective quality assessment experiment, carried out on Holografika's Holovizio HV721RC light field display [3]. The authors generated their own stimuli by capturing 3 different objects using the turntable approach, and converting the datasets to the display's 50-degree FOV. A single virtual object was created as well, whose rendering parameters matched the capturing parameters of the real objects. The visualized content was assessed by 20 test participants - naïve subjects - from multiple (5) fixed positions, at a distance of $150 \mathrm{~cm}$. As the height of the screen was $88 \mathrm{~cm}$, this length corresponded to a viewing distance of $1.7 \mathrm{H}$. The assessment task was to rate the visual quality on a continuous scale ranging from 1 to 5 , where 1 corresponded to "poor" and 5 to "excellent" quality; which can be simply considered to be the continuous version of the 5-point Absolute Category Rating (ACR) scale [4]. The angular resolution was at a fixed value throughout the entire experiment ( 1 view per degree), but several types of distortions (Gaussian noise, Gaussian blur, etc.) were applied to the content. The acquired subjective scores were used to validate a full-reference (FR) 3D objective quality metric proposed by the authors, composed of a spatial and an angular component. The work emphasizes the importance of horizontal parallax; however, even though test participants moved between viewing positions, each and every test stimulus was observed and assessed from a fixed position. They did experience the visual phenomenon of horizontal parallax, but the observed visual quality between viewing positions was not to be evaluated. Of course the parallax effect is present even in case of a fixed-position observer - due to the distance between the eyes and the horizontal movements of the head, not to mention the depth cues observed by a single motionless eye - but the test participants' visual experience of parallax is more intense during actual movement. One could argue that the movement phase during the experiment must have affected the assessment at the given fixed position; however, the work does not specify the form of movement (i.e., sideways movement facing the screen of the display with uninterrupted visual attention) for all test participants, as visual experience between the positions of observation was not considered in the rating task.

Using the same LFD as in the previously introduced work, Vamsi et al. evaluated the effectiveness of a live capture system proposed by the authors [5]. The capture on the front end of the system was performed by an array of 18 web cameras, each connected to a PC node, and each node rendered images for 4 optical modules on the back end. In a different work, Vamsi et al. conducted a subjective performance assessment experiment on direct 3D interaction [6]. The authors used a Leap Motion Controller to track the free-hand gestures of the test participants during the experiment, in which they had to touch given red surfaces in a scene visualized by the LFD. The positions of the surfaces were chosen in a way to cover the entire FOV. The virtual scene was displayed by a small projection-based LFD, developed by Holografika. The interactive task - measuring task completion time, cognitive workload and perceived user experience - compared 2D and 3D scenarios, and was performed by 12 test participants. Due to the type of the task, the test participants observed the visualized scenes from an arm's distance. The findings indicate high efficiency and intuitiveness, reflected from the negligible difference between the cognitive workloads of $2 \mathrm{D}$ and 3D tasks, however, the 3D LFD-visualized scenario also had a higher average task completion time requirement. The perceived quality was measured through a User Experience Questionnaire (UEQ) [7], collecting data in the aspects of "Attractiveness", "Perspicuity", "Efficiency", "Dependability", "Stimulation" and "Novelty". Even though the 3D scenario achieved a high performance in scores, beside the feeling of novelty - due to the novel nature of this new visualization technology - no major difference was found in the investigated aspects of user experience.

The subjective evaluation performed by Dricot et al. [8] addresses the feasibility of a light field video service with 80 views in an approximately 40-degree FOV, resulting in 2 views per degree angular resolution of the $3 \mathrm{D}$ visualization. The LFD used in the experiment was Holografika's Holovizio C80 cinema system [9]. In order to generate the stimuli, the authors used a linear camera system that consisted of 80 cameras, so one camera captured one view. Rendered stimuli were created as well, with a corresponding virtual camera system of 80 cameras in a linear setup. Using an arc camera layout would have been possible as well, but different camera system setups have significantly different coding performance, and as the aim of the subjective studies was to evaluate and compare coding configurations, only linear camera arrangement was used. During the encoding process, specific views were skipped, which did not result in angular resolution drop as they were synthesized, but degraded visual quality and introduced artifacts. The authors used the Double Stimulus Impairment Scale (DSIS) [10] method to assess the impairments of the visual stimuli, caused by compression and synthesis. A total of 16 test participants completed the rating task of the experiment - a combination of naïve and expert subjects - who viewed the screen of the display from a distance of 6 meters (roughly $3.3 \mathrm{H}$ based on the system specifications). The test participants viewed the visualized content from fixed positions, between 1 and 6 test participants per session. The work also particularly addressed horizontal motion parallax in a session where test participants moved 2 meters sideways during video stimulus playback. The authors conclude that the perception of horizontal motion parallax was not affected by specific compression artifacts, only rather by the variations of severe artifact along the viewing angle, which means that viewing the impaired stimulus from a given position had undisturbed horizontal parallax, but the introduction of movement to the same subjective evaluation degraded the smoothness of the parallax effect. Note that for test cases with a low extent of visual impairments (rated as "Imperceptible" or "Perceptible but not annoying" on the scale) the movement of the test participant did not disturb the horizontal parallax effect. The results of this research indicate that the horizontal parallax is easier to disturb during observer movement than during fixed-position observation, thus the exclusion of movement allows higher tolerance towards quality degradations.

The experiment of Kara et al. [11] used the same LFD (Holovizio C80 cinema system [9]) as the previously explained work. The research investigated the effect of different angular resolutions on the perceived quality. The number of views ranged from 15 to 150 , meaning at least 0.3 and at most 3.3 views per degree as angular resolution. The test stimuli were rendered using a linear array of virtual cameras. The subjective 
assessment task was completed by 20 test participant - naïve subjects - who had to move 2 meters sideways during content visualization and rate the quality on a discrete scale from 1 to 10 . The obtained results show that an angular resolution of 2 views per degree or more can provide sufficient quality to maintain a good visual experience, and that the disturbance of horizontal parallax severely penalizes scores on lower angular resolutions. The authors also point out the possible distortions and inconsistencies in rating scores due to the novel nature of the visual phenomenon of reduced angular resolution.

In all three works with content visualization, content dependency of the obtained results was apparent, commonly relating to depth values and model complexity. In our research, we used test stimuli diverse in both aspects. We chose source contents that had parameters similar to the models used in recent related work, and also generated stimuli that were very sensitive to any impairment of $3 \mathrm{D}$ visualization, due to large depth and high model complexity. As declared in the research question, the experiment presented in this paper involves only fixed-position observers, in order to investigate the increased perceptual tolerance on the horizontal parallax effect of a cinema-like use case scenario.

\section{EXPERIMENTAL SETUP}

\section{A. Display and environment}

In the experiment, we used Holografika's Holovizio C80 cinema system [9], which is a projection-based light field display with a screen width of $3 \mathrm{~m}$ and a height of $1.85 \mathrm{~m}$. The brightness of the screen was $1500 \mathrm{~cd} / \mathrm{m}^{2}$, and it was calibrated to enable the $3 \mathrm{D}$ visualized content to be observable in a 45 degree FOV.

The subjective assessment task took place in a closed laboratory environment, parameters of which were chosen to simulate the conditions of a cinematic experience. The room had lighting conditions of approximately $8 \mathrm{~lx}$, as there was no light source other than the LFD itself, which on its own provided sufficient illumination for the test participants to fill the rating sheets.

A test participant was allocated to a given seat during the subjective test; switching seats or leaving the seat was not possible. There were in total 6 seats available in the experiment, in 2 rows of 3 (see Figure 1). During the experiment, participants were uniformly distributed to the seats, which means that all seats were occupied by the same number of observers throughout the series of tests. The first row of seats was $4.6 \mathrm{~m}$ away from the screen of the display, followed by the second row at $5.6 \mathrm{~m}$. Based on the physical parameters of the screen (height of approximately $1.85 \mathrm{~m}$ ), this corresponds to a $2.5 \mathrm{H}$ and a $3 \mathrm{H}$ distance, according to the recommendations [12]. The seats also had a sideways distance of $1 \mathrm{~m}$ between them.

\section{B. Test case parameters}

The only variable in the test cases was the angular resolution. As the FOV was a fixed 45 degree, the number of views allocated to the optical engines was changed. As prior experimental results determine 2 views per degree - 90 views in this case - to provide sufficient perceptual quality,

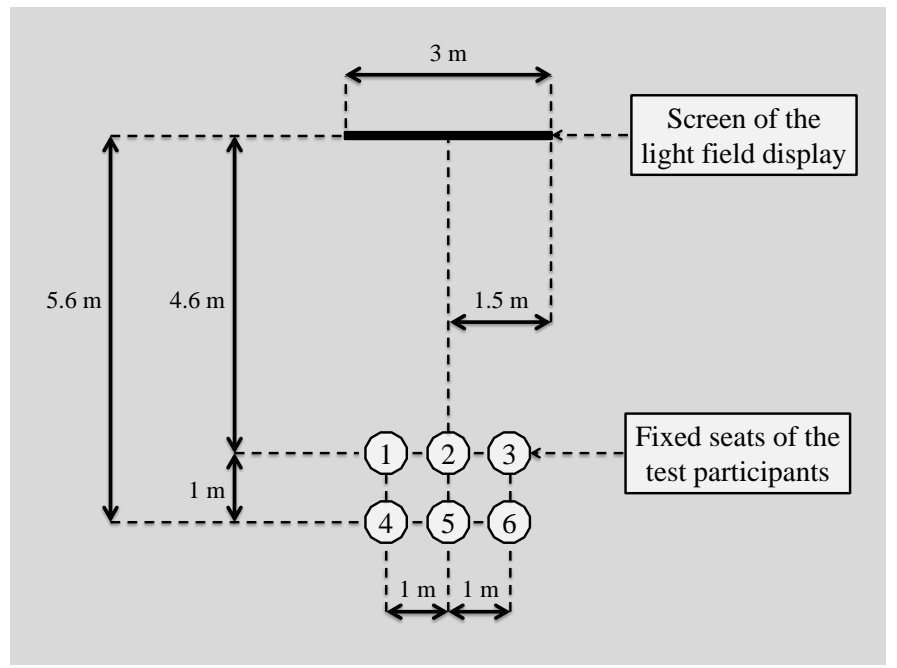

Fig. 1. Experimental setup of the cinema environment.

we decided to test angular resolutions particularly below this limit. We defined a total of 10 test cases plus a reference with separate angular resolutions. The reference - which was not be assessed and was not present as a hidden reference - had 90 views, this was reduced by steps of 10 until $45(80,70,60$, 50 and 45), and went even below that by steps of 5 until 20 $(40,35,30,25$ and 20). We decided to choose a finer grain for the test cases with angular resolution below 1 view per degree, as the difference on such levels result in a greater change of perceived quality [11].

\section{Scales of the measurement}

For each and every test case, participants had to evaluate quality on 2 scales. One was a 25-point quasi-continuous scale [4] for the perceived quality. We considered to use a 5point ACR scale [4]; however, using a fine-grained scale was preferable, in order to be able to compare the small differences in quality between given the test cases. Instead of choosing a larger scale like an ACR running from 0 to 10 [4], we selected the visual approach of the quasi-continuous scale. Due to the fact that the size of the scale was not apparent to the test participants, visual decisions were made instead of numerical ones. Using such scale also reduces the bias originating from the interpretation of the labels, and allows data to be collected without ambiguity. As the decreasing number of views in the FOV degrades the visual experience through perceptual phenomena such as the cross-talk effect, visible edges of discrete views or sudden "jumps" in horizontal motion parallax, a Degradation Category Rating (DCR) scale [4] was considered as well, and could have been suitable, but the clustered decisions (detectable or not, annoying or not, annoying to what extent) were unnecessary due to the coexistence of a different scale in the experiment.

The other one was a binary scale [4] for quality acceptance. We found this scale particularly important for the research, as such a scale not only clarifies the data collected by the other scale - e.g., a 15 out of 25 can be interpreted completely differently for two test participants - but also reports on the final judgment of the user regarding the quality of the displayed content. In practice, at the end of the day, most of the active 


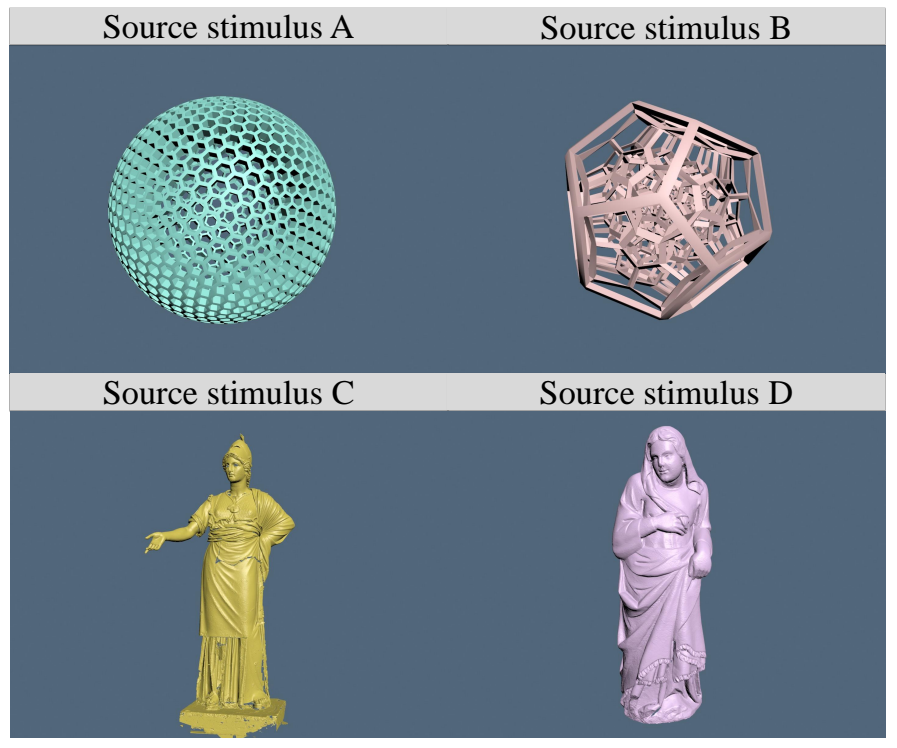

Fig. 2. Source stimuli of the experiment.

user decisions are simply binary (e.g., buying a display or not). In the case of our experiment, the test participant decided whether he or she found the perceived quality acceptable or not.

\section{Displayed source content and test structure}

The 10 test cases were applied to 4 different source contents (see Figure 2), so each and every test participant had to provide ratings 40 times on 2 scales. All visual stimuli were rendered using a linear setup of virtual cameras, generating visual contents directly in the desired number of views, in a fixed spatial resolution of $1440 \times 1080$. Although spatial resolution does affect the perceived quality on LFDs [13], its effect on the Human Visual System (HVS) is marginal between higher resolutions [14].

The reason why we used only rendered visual content was the ease of stimuli generation with an arbitrary angular resolution. Accomplishing the corresponding task with a real linear camera setup would have either required a significant effort to realign the cameras for each and every different angular resolution, or skipping intermediate views from the recorded visual dataset, which can be imprecise in certain cases.

Stimulus A and B were complex mathematical bodies with large depths [15], stimulus C and D were laser-scanned statues with smaller depths [16]. The difference between A and B was that while A (polyhedron with 972 faces) had a detailed, uniform grid on the front (closest to the observer), stimulus B (structure of 120 regular dodecahedra) had a simple, smooth surface segment on the side of the object viewed from the observer, thus we expected stimulus A to be more sensitive to the reductions in angular resolution - even though the center of the body was much more detailed. In general, both A and B suffered significant penalties in the perceived visual quality, even at the highest angular resolutions included in this experiment. The statue of stimulus $\mathrm{C}$ had its right hand closer to the observer (compared to the rest of the model), while stimulus D was more compact. However, there were bigger depth differences in the lower half of the model of stimulus D.

Each stimulus was displayed for 10 seconds, followed by 5 seconds of blank screen - containing only the background color - during which participants rated the given stimulus. The total duration of the assessment task was approximately 12 minutes, and it was uninterrupted, without breaks.

As the test participants were all new to such displays (naïve subjects), they were not familiar with the visual phenomenon of reduced angular resolution, which can result in distortions and inconsistencies of the subjective test results [11]. Beyond the regular learning phase of QoE experiments, test participants were particularly introduced to angular resolution prior to the assessment task, using a set of simple shapes (plain-color cubes with different hues) covering a large depth, rendered in different angular resolutions.

\section{E. Test participants}

A total of 22 test participants completed the quality assessment task, with an average age of 31 . From these observers 18 were male and 4 were female. As test participants were uniformly distributed among the 6 seats in the experimental setup of the light field cinema, the visualized content was assessed 4 times from position 1, 2, 3 and 4 and 3 times from position 5 and 6 (see Figure 1). Taking observer positions into consideration is beyond the scope of this paper, but further extension of the research will aim to have an equal number for all positions.

\section{RESULTS}

This section of the paper presents the analysis of the subjective scores obtained during the experiment. Mean results for all test conditions and per-stimulus ratings are detailed in this paper, but in-depth pattern analysis, correlation calculations and certain additional result separations - such as based on sideways observer position and distance from the screen - are to be included in further publication of the extended experimental results.

\section{A. Overall analysis}

The scores collected on the 25-point quasi-continuous scale can be looked at as results on a 5-point ACR scale, if we allocate the intervals to the values -1 to 5 corresponds to "bad, 6 to 10 to "poor", 11 to 15 to "fair", 16 to 20 to "good" and 21 to 25 to "excellent". This approach of data mapping is not recommended for a precise analysis of the results, as the ratings collected by these 2 scales cannot be accurately matched in such a way due to several reasons; however, it provides an initial understanding of the scores.

The mean scores of perceived quality (see Figure 3 ) suggest that not even the test case with the highest number of views (80) managed to fully satisfy the test participants on average. Indeed, as reported by a similar research [11], perceivable differences can be found above 2 views per degree, which result in a more realistic, natural sight and thus a better user experience. The mean degradation in scores appears to have a linear relation with the selected angular resolution values; note 


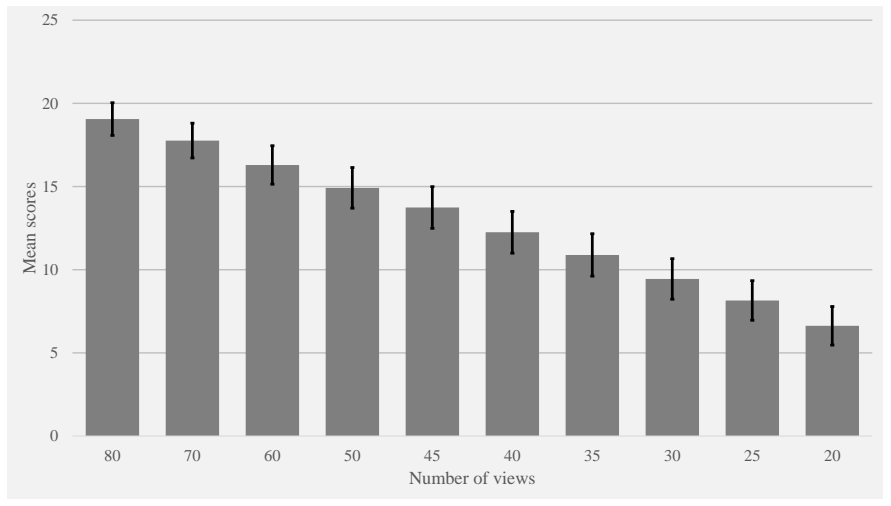

Fig. 3. Overall perceived quality.

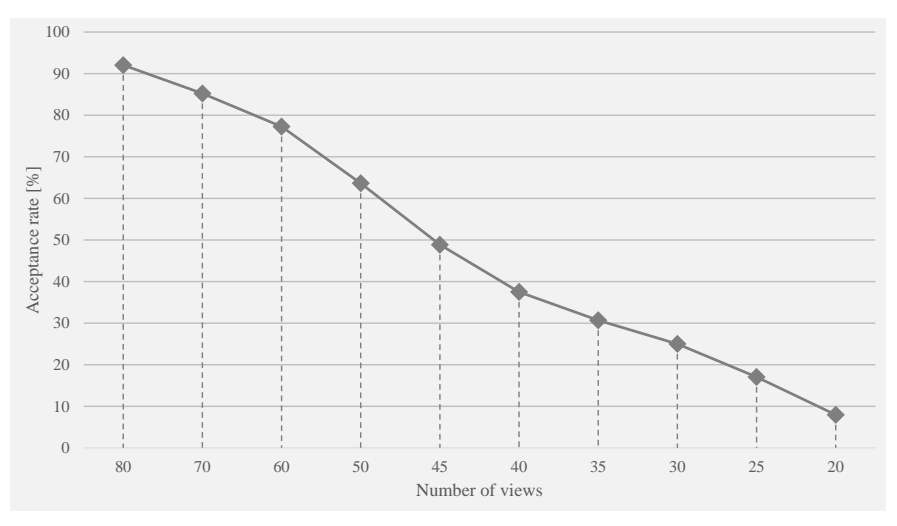

Fig. 4. Overall quality acceptance.

that the number of views is not reduced in a uniform manner for the whole test, as explained in the previous section. Based on these ratings, the angular resolution of approximately 1 view per degree obtained mean scores in the middle interval of perceived quality, which can be considered to be adequate in general. After all, according to the recent search of Tamboli et al. for suitable free-viewpoint visual contents for LFD experiments [2], most of the publicly available datasets were captured with an even lower angular resolution.

Even though the lowest angular resolutions in this experiment (around 0.5 views per degree) provide a severely distorted view of the objects, their mean scores did not go down to the bottom interval of scores. The so-called "jumps" in horizontal motion parallax have their highest level of effect on the perceived quality during motion, and motion was excluded from this experiment, so the degradation in scores was mainly due the cross-talk effect. Tamboli et al. are also perfectly correct when they state that "most of the subjective studies were conducted with static observers, from a single viewpoint in front of either a stereoscopic or an auto-stereoscopic display. Such approaches fail to leverage the unique capability of super-multiview displays of rendering 3D content with continuous horizontal parallax." [2]. Without a doubt, static, fixed-position observers cannot experience the full extent of horizontal motion parallax, but because of this, they are also less prone to be affected by the disturbances in the parallax effect.

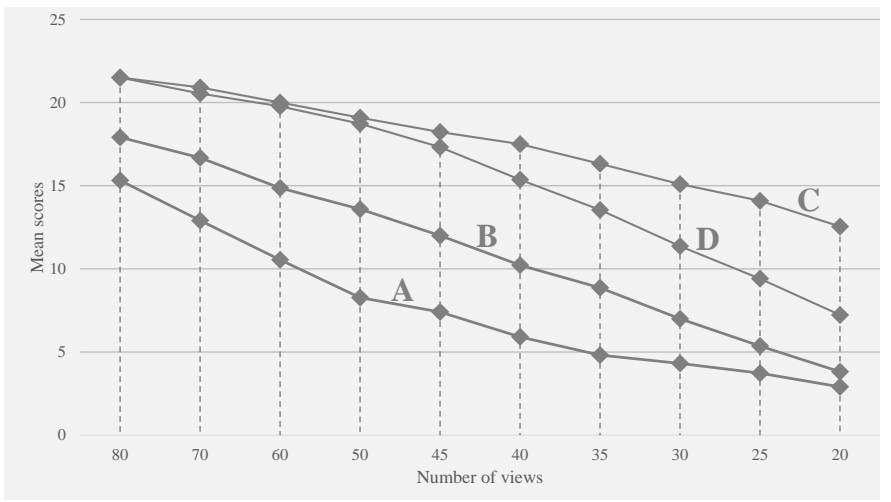

Fig. 5. Quasi-continuous scores of the stimuli.

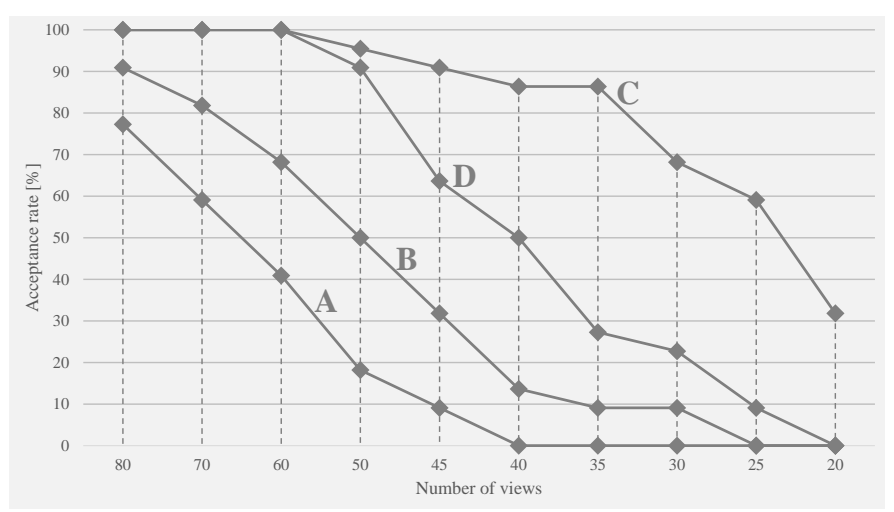

Fig. 6. Binary acceptance scores of the stimuli.

Similar tendencies can be observed in the overall scores of quality acceptance (see Figure 4), which reports the percentage of test subjects who found the provided quality acceptable. The acceptance rate of the test case with roughly 1 view per degree angular resolution is around 50 percent. For test conditions with the most reduction in angular resolution, acceptance goes even below 10 percent, understandably.

\section{B. Quasi-continuous ratings}

If we examine the different source stimuli separately and compare their mean scores on perceived quality (see Figure 5), first we see that $C$ and $D$ performed significantly better than $A$ and $\mathrm{B}$ (as expected). The rating tendencies for the same model types are similar, but certain differences can be spotted, due to their constructions. From the first two, B performs better, and from the second pair, $\mathrm{C}$. On the higher and middle range angular resolutions, there are bigger differences for the first pair, and become similar on the lowest ones. The opposite can be seen for the second pair, as significant differences in perceived quality appear on the lowest angular resolutions.

\section{Binary acceptance ratings}

The previous statements comparing the two model types are similarly valid for the acceptances rates (see Figure 6); however, differences are more intense in general, and we can clearly observe the effect of the model parameters. For A, the initial acceptance rate is low already - compared to $\mathrm{B}$ and especially to $\mathrm{C}$ and $\mathrm{D}$ - but drops to zero below 


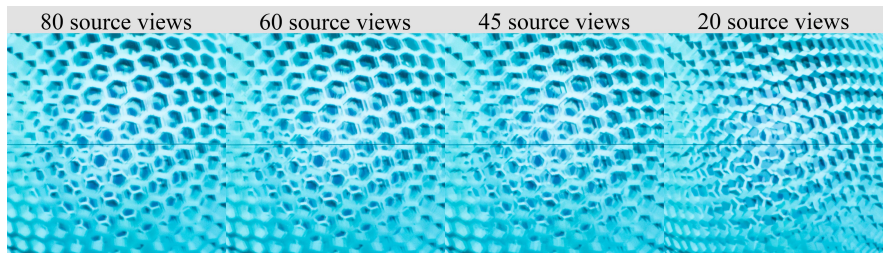

Fig. 7. Different angular resolutions for stimulus A, captured by a single camera from position 2 .

1 view per degree angular resolution, due to the cross-talk effect on the front grid; below 60 views, elements of the grid start overlapping (see Figure 7). For B, degradation is more graceful, but also becomes unacceptable when the smaller details in the core of the model become indistinguishable below 1 view per degree angular resolution. For both $\mathrm{C}$ and D, the 3 highest angular resolutions received complete acceptance, the real differences surface below 50 views. In case of stimulus $\mathrm{C}$, until 35 views, the main issue with the visuals is the outreaching arm of the statue, but the rest of the model is nearly completely unaffected. In the mean time, stimulus D received a very low acceptance for the same value, and actually reaches zero at the lowest angular resolution.

Results on both scales indicate high content dependency, as also seen in related works. Subjective sensitivity towards the perceived quality of visualization fundamentally depends on depth values and structural complexities of closer elements. The best portrayal of content dependency is the acceptance rate at 45 views (see Figure 6), as the results cover almost the entire $\mathrm{Y}$ axis in a near-to-uniform manner. We conclude that on average 1 view per degree is a fair lower bound for $3 \mathrm{D}$ visualization on LFDs, but the double of that is preferred, and excellent quality - and thus the best user experience - necessitates an even higher angular resolution for certain contents. Of course this only applies to use case scenarios which include static, fixed-position observers. In case of moving observers e.g., 3D visualization at a public exhibition - higher angular resolutions are required in order to achieve smooth horizontal motion parallax.

\section{CONCLusion}

In this paper, we presented the results of a subjective quality assessment experiment on the angular resolution of light field displays. The use case scenario was a cinema-like environment, with a large light field cinema, dark surroundings and observers sitting in given positions. Our results indicate that the lack of user movement allows tolerance towards angular resolutions below 2 views per degree, depending on the visualized content. As static observers do not experience the full capabilities of such displays, their perception of the horizontal parallax effect is also less disturbed when visualization consists of less views in a given field of view.

In future works, we intend to extend our currently available results with the ratings of more test participants, perform comparisons between static and moving observers, and further study the notion of angular resolution and the parallax effect. A more accurate understanding of the requirements for angular resolution shall allow a cost-effective transmission of data for light field multimedia services.

\section{ACKNOWLEDGMENT}

The work in this paper was funded from the European Union's Horizon 2020 research and innovation program under the Marie Sklodowska-Curie grant agreement No 643072, Network QoE-Net. The work in this paper was also funded from the European Union's Horizon 2020 research and innovation program under the Marie Sklodowska-Curie grant agreement No 676401, Network ETN-FPI.

\section{REFERENCES}

[1] P. T. Kovács, K. Lackner, A. Barsi, Á. Balázs, A. Boev, R. Bregović, and A. Gotchev, "Measurement of perceived spatial resolution in 3D light-field displays," in International Conference on Image Processing (ICIP). IEEE, 2014, pp. 768-772.

[2] R. R. Tamboli, B. Appina, S. Channappayya, and S. Jana, "Supermultiview content with high angular resolution: 3D quality assessment on horizontal-parallax lightfield display," Signal Processing: Image Communication, vol. 47, pp. 42-55, 2016.

[3] Holovizio 721RC light field display, www.holografika.com/Documents/Holovizio_721RC-emailsizenomovie.pdf (retrieved Jan. 2017).

[4] ITU-T Rec., "P.910: Subjective video quality assessment methods for multimedia applications," 2008.

[5] V. K. Adhikarla, F. Marton, T. Balogh, and E. Gobbetti, "Real-time adaptive content retargeting for live multi-view capture and light field display," The Visual Computer, vol. 31, no. 6-8, pp. 1023-1032, 2015.

[6] V. K. Adhikarla, J. Sodnik, P. Szolgay, and G. Jakus, "Exploring direct $3 \mathrm{~d}$ interaction for full horizontal parallax light field displays using leap motion controller," Sensors, vol. 15, no. 4, pp. 8642-8663, 2015.

[7] B. Laugwitz, T. Held, and M. Schrepp, "Construction and evaluation of a user experience questionnaire," in Symposium of the Austrian HCI and Usability Engineering Group. Springer, 2008, pp. 63-76.

[8] A. Dricot, J. Jung, M. Cagnazzo, B. Pesquet, F. Dufaux, P. T. Kovács, and V. K. Adhikarla, "Subjective evaluation of super multiview compressed contents on high-end light-field 3D displays," Signal Processing: Image Communication, vol. 39, pp. 369-385, 2015.

[9] Holovizio C80 light field cinema system, www.holografika.com/Documents/HoloVizio_C80.pdf (retrieved Jan. 2017).

[10] ITU-R Rec., "BT.500-13: Methodology for the subjective assessment of the quality of television pictures," 2012.

[11] P. A. Kara, M. G. Martini, P. T. Kovács, S. Imre, A. Barsi, K. Lackner, and T. Balogh, "Perceived quality of angular resolution for light field displays and the validity of subjective assessment," in International Conference on 3D Imaging (IC3D). IEEE, 2016.

[12] ITU-T Rec., "BT.2021: Subjective methods for the assessment of stereoscopic 3DTV systems," 2015.

[13] P. T. Kovács, A. Boev, R. Bregović, and A. Gotchev, "Quality measurements of 3D light-field displays," in Proc. Eighth International Workshop on Video Processing and Quality Metrics for Consumer Electronics (VPQM), 2014.

[14] P. A. Kara, P. T. Kovács, M. G. Martini, A. Barsi, K. Lackner, and T. Balogh, "Viva la resolution: The perceivable differences between image resolutions for light field displays," in 5th ISCA/DEGA Workshop on Perceptual Quality of Systems (PQS), 2016, pp. 107-111.

[15] George W. Hart's Rapid Prototyping Web Page, www.georgehart.com/rp/rp.html (retrieved Jan. 2017).

[16] Jotero.com 3D-Scan and 3D Measurement, forum.jotero.com/viewtopic.php?t=3 (retrieved Jan. 2017). 